\title{
Hyperspectral Holography and Laser Diffractometry of Erythrocytes
}

\author{
Andrei E. Lugovtsov ${ }^{1,2^{*}}$, Vladislav D. Ustinov ${ }^{3}$, Georgy S. Kalenkov ${ }^{4}$, Sergey G. Kalenkov ${ }^{5}$, \\ and Alexander V. Priezzhev ${ }^{1,2}$ \\ ${ }^{1}$ Department of Physics, M.V. Lomonosov Moscow State University, 1 Leninskie Gory, Moscow 119991, Russia \\ ${ }^{2}$ International Laser Center, M.V. Lomonosov Moscow State University, 1 Leninskie Gory, Moscow 119991, Russia \\ ${ }^{3}$ Moscow Center of Fundamental and Applied Mathematics, M.V. Lomonosov Moscow State University, 1 Leninskie \\ Gory, Moscow 119991, Russia \\ ${ }^{4}$ Institute of Geosphere Dynamics (IDG RAS), 38/1 Leninskiy prospekt, Moscow 119334, Russia \\ ${ }^{5}$ Moscow Polytechnic University, 38 Bolshaya Semyonovskaya str., Moscow 107023, Russia \\ *e-mail: anlug@biomedphotonics.ru
}

\begin{abstract}
Geometrical 3D models of human red blood cells in dry smear were obtained experimentally using hyperspectral holography technique. Corresponding diffraction patterns in the far field diffraction zone were calculated. Visibility values of the diffraction patterns were obtained in cases of low and high red cell distributions width (RDW). Our study reveals that although the visibility is influenced by the cells shapes, this parameter can still be used to assess red cell distribution width in clinical practice. (C) 2020 Journal of Biomedical Photonics \& Engineering.
\end{abstract}

Keywords: laser diffractometry; hyperspectral holography; erythrocyte; visibility.

Paper \#3368 received 22 May 2020; revised manuscript received 18 Jun 2020; accepted for publication 18 Jun 2020; published online 24 Jun 2020. doi: 10.18287/JBPE20.06.020306.

\section{Introduction}

Modern medical diagnostics is based on blood tests that allow one to insure diagnosis with high accuracy. Red cell distribution width (RDW) is known as a crucial parameter in case of blood anemia. RDW is a measure of how much the erythrocytes differ from each other in size. RDW is close to zero, when all cells have similar size, and increases when the sizes are different. RDW has been evaluated for decades, and its application field grows rapidly. Recent investigation performed with more than 8000 people shows that RDW serves as a reliable mortality predictor independent of the disease type, see Ref. [1]. Thus, accurate diagnostic instruments for measuring this parameter are in high demand.

There is a number of different methods to measure RDW, each of which has its own advantages and disadvantages [2]. For example, optical microscopy enables one to see the erythrocytes and estimate their sizes visually. This is the most common method and it allows one to investigate the erythrocytes shapes by looking at the image. However, only a few hundreds of cells can be analyzed during a short time. The Coulter cell counter measures about 10000 cells in a few seconds. However, it should be assumed that the shape of the cells is spherical that leads to certain misinterpretation of the results. Thus, more reliable and efficient RDW measurement techniques are still required.

In this article, we continue our research on laser diffractometry of erythrocytes, which has been started by our group several years ago. In this technique, erythrocytes are illuminated by a laser beam and a diffraction pattern is observed in the far-field diffraction zone. One can solve integral equation in which the diffraction pattern is used as an input data and the solution is erythrocyte size distribution [3]. Another strategy is to rely on certain features of the diffraction pattern. For example, the visibility of the pattern reflects the shape and size of particles, see Ref. [4].

In Refs. [5-6], it was shown that the visibility of the diffraction pattern monotonically depends on RDW of the cells in the given blood sample. Thus, laser diffractometry allows one to assess the value of RDW. It is possible to illuminate hundreds of thousands of cells within a moment. Calculation of the visibility parameter can also be performed during less than a second using any modern personal computer. This 
makes the method more preferable to others. However, the shape of the cells remains unknown as in the Coulter counter and one has to use some 3D geometrical model of the cells.

In hyperspectral holography $(\mathrm{HH})$, one obtains the phase modulation of the light rays passing through the object with resolution up to half of the incident light wavelength. In previous Refs. [7-8], HH technique has been well developed and successfully tested on biological particles. In the present work, we have applied $\mathrm{HH}$ to measure the phase profiles of red blood cells on a glass smear. This enables one to reconstruct the 3D geometrical model of the erythrocytes shape. Although we use the specific technique developed in Refs. [7, 8], one can find other works on the topic, see, for example, Refs. [9, 10].

The main aim of this paper is to enhance laser diffractometry of erythrocytes by using the 3D geometrical model of real cell experimentally obtained using $\mathrm{HH}$ method. The visibility of the diffraction pattern depends not only on RDW value but also on the model of the cells shape. In this paper, we validate the dependency of the visibility parameter on RDW comparing different theoretical and experimentally obtained cells models.

Dry blood smears are used worldwide in routine medical tests. There are techniques that enable one to study cells in their original wet environment. In this paper, we consider dry smears in order to be closer to real medical practice.

\section{Materials and Methods}

The 3D geometrical model was obtained experimentally by using HH method as shown in Fig. 1. The method is described in a separate paper [7]. We have drawn the blood samples from a healthy male donor 35 years old. The dry blood smear was prepared according to a conventional medical protocol. In Fig. 1, the object was an erythrocyte on the glass smear located separately from other cells. The cell can change its normal shape of the biconcave disk during drying. Collecting data from the $\mathrm{HH}$, we were be able to estimate this change precisely.

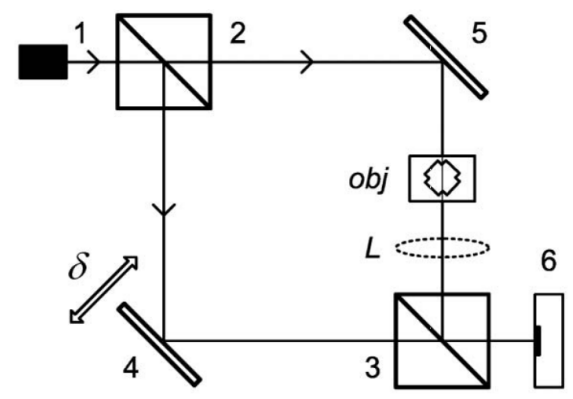

Fig. 1 Principal scheme of the interferometer for hyperspectral holograms registration. Polychromatic light source 1; beam splitter cubes 2, 3; mirrors 4, 5; CCD/CMOS sensor 6 ; obj - object under the study (erythrocyte); L - objective. See Ref. [7] for more details.
The collected data were used to model laser light scattering by erythrocytes in laser diffractometer. Principal scheme of this device is shown in Fig. 2, see Ref. [6] for more details.

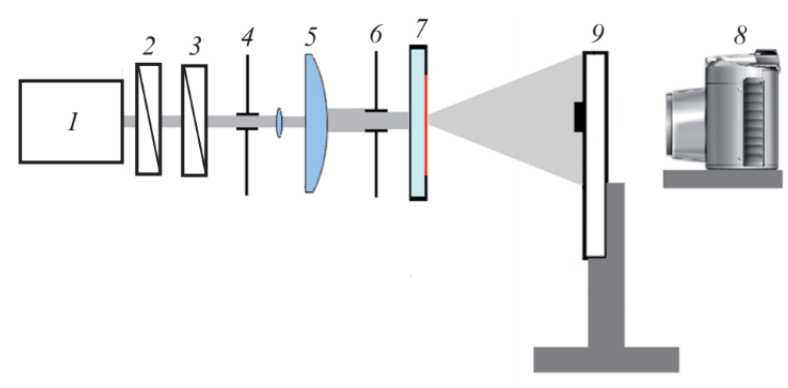

Fig. 2 Laser diffractometer; 1 - He-Ne laser; 2, 3 polarizers; 4, 6 - apertures; 5 - lens system; 7 - blood smear; 8 - camera; 9 - translucent screen with a laserbeam absorbing plate. See Ref. [6].

Laser beam with wavelength $0.63 \mu \mathrm{m}$ illuminates the cells on the smear 7 . The laser power is controlled by the polarizers. The beam width is controlled by aperture and telescope. The diffraction pattern is detected by the camera on the observation screen. The key points are as follows:

- typical size of erythrocytes is equal to $7.5 \mu \mathrm{m}$ and is about one order of magnitude higher than the laser wavelength, which insures that forward scattering in Mie regime occurs;

- the light scattering is single due to low concentration of cells, which insures that they do not overlap with each other;

- the observation screen is placed in the far-field diffraction zone.

$\mathrm{He}-\mathrm{Ne}$ lasers yield a higher visibility of diffraction pattern as compared to a LED sources. Calculation of light scattering by single particle was performed by means of anomalous diffraction approximation. In this approximation, we assume that each ray passing through particle does not refract but gets additional phase modulation. Note, that only forward scattering with small scattering angles up to $15^{\circ}$ are computable with high accuracy using this approximation. See Ref. [11] for more details on the application of the anomalous diffraction approximation for laser diffractometry of erythrocytes. The formula for electrical field of scattered light by single particle is as follows:

$$
\begin{aligned}
& -\frac{i e^{i k l}}{\lambda l} \iint_{S} E_{0} \cdot e^{-\frac{i k}{l} x \cdot x_{0}-\frac{i k}{l} y \cdot y_{0}} \times \\
& \times\left(e^{-i k n_{0}\left(\frac{n_{1}}{n_{0}}-1\right) \cdot h(x, y)}-1\right) d x d y= \\
& =E\left(x_{0}, y_{0}\right),
\end{aligned}
$$

where $\mathrm{S}$ is the area bounded by the particles shadow in the laser beam; $k=2 \pi / \lambda$ - wavenumber of incident light, $\lambda$ - laser wavelength in vacuum, $n_{0}-$ absolute refractive index of the medium (absorption is 
neglected), $n_{1}$ - absolute refractive index of the particle. Point $(x, y)$ goes through the area $S$, point $\left(x_{0}, y_{0}\right)$ is fixed on the observation screen. Value $h(x, y)$ determine the particle thickness along the direction of laser beam propagation. Parameter $l=\sqrt{z^{2}+x_{0}^{2}+y_{0}^{2}}$ is distance from the point $(0,0)$ on the site $S$ to the point $\left(x_{0}, y_{0}\right)$ on the observation screen, $z$ - distance between the sample and the observation screen. $E_{0}-$ constant electric field complex amplitude of a plane incident wave. $E\left(x_{0}, y_{0}\right)$ - the desired complex amplitude of the electric field of the wave scattered by a given single particle.

Note that in laser diffractometry one obtains only the intensity of the scattered light $I\left(x_{0}, y_{0}\right)=\left|E\left(x_{0}, y_{0}\right)\right|^{2}$ measured by the camera at the observation screen. We refer to the $2 \mathrm{D}$ function $I\left(x_{0}, y_{0}\right)$ as a diffraction pattern. In our model, we assume that each cell has some known shape given by the function of cell thickness $h(x, y)$ in Eq. (1). We use random number generator to place cells uniformly on the plane. We calculate the integral in Eq. (1) for each particle and sum up the results according to the interference of rays coming from different cells to the same points on the observation screen. This enables one to calculate the diffraction pattern $I\left(x_{0}, y_{0}\right)$ at each point. As the cells have random coordinates, this diffraction pattern has some speckles corresponding to the coordinates and amount of the cells but not their shape geometry.

In the case of an ideal theoretical model, the area $S$ in Eq. (1) is a circle with a given diameter. Otherwise, $S$ is determined by calculating the particle contour from the given experimental $\mathrm{HH}$ image. In this case, the size of $S$ is determined as the diameter of a circle with equivalent area. The histogram of the cell sizes represents the function of particle size distribution. In this paper, we assume it to be uniform on some physically meaningful fixed segment. We treat RDW as the standard deviation of the cell size distribution divided by its mean diameter.

Let us introduce visibility $v$ of a diffraction pattern. Fig. 3 helps to explain the situation. Consider the diffraction pattern calculated as described above. As shown in Fig. 3(a), this pattern is non-symmetric because the cells may have irregular shapes and random coordinates. Consider polar coordinates $(r, \varphi)$ on the observation screen. Let us average the intensities at each polar radii $r$ over all angles $\varphi: I(r)=\frac{1}{2 \pi} \int_{0}^{2 \pi} I(r, \varphi) d \varphi$. This procedure enables one to treat all directions in the diffraction pattern in equivalent way. Function $I(r)$ contains speckles coming from the interference of laser light scattered by different particles with arbitrary positions inside of the laser beam. In order to overcome their influence, we calculate a polynomial of degree 10 best fitting the function $I(r)$ in the segment close to the first interference fringe of the diffraction pattern. In the first dark fringe $I(r)$ has a local minimum and in the first bright fringe $I(r)$ has a local maximum. Denote them by $I_{\min }$ and $I_{\max }$ respectively. Let visibility be $v=\left(I_{\max }-I_{\min }\right) /\left(I_{\max }+I_{\min }\right)$ by its definition.

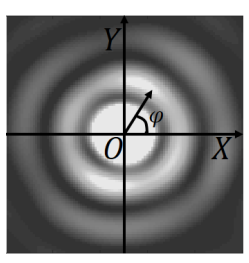

(a)

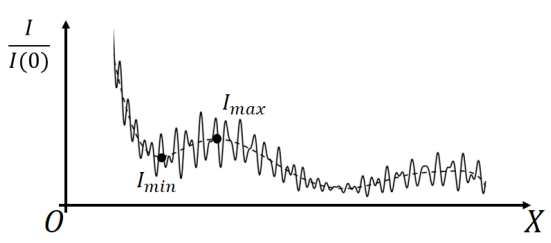

(b)
Fig. 3 Part (a) shows diffraction pattern that corresponds to some non-symmetric cell image. Both Cartesian and polar coordinates are pictured. In part (b), solid line is the function of the intensity after the averaging procedure and the dashed line is the best fitting polynomial. $I_{\max }$ and $I_{\min }$ are values of first bright and dark interference fringes. One can see them in (a) near the center.

\section{Results}

Fig. 4(a) shows a typical phase image of red blood cell obtained by the HH method. An automatically extracted contour of a cell is shown in Fig. 4(b). One can see that the cell is non-symmetric that should result in a nonsymmetric diffraction pattern. If the cell was an ideal cylinder with a round base, then the visibility of the diffraction pattern would be exactly 1 . Even if the cell had a shape of an ideal biconcave disk, the visibility would equal about 0.86 . However, our calculations show that for such a particle as in Fig. 4, the visibility is about 0.5 . This is mainly due to the contribution of phase non-symmetry inside the particle, and not to its contour. For example, if we set the phase inside of the contour to be constant, the visibility for a single particle remains equal about 0.98 .

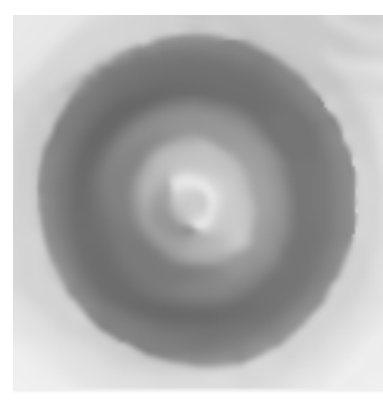

(a)

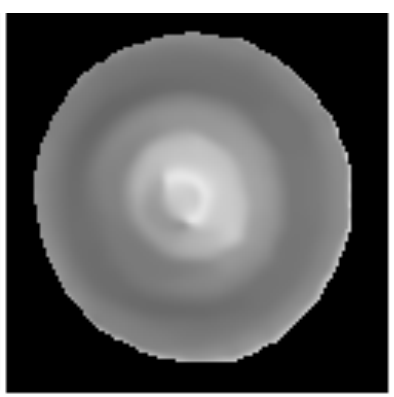

(b)
Fig. 4 Part (a) shows the phase image of a typical cell obtained by the HH method. Part (b) shows a result of contour recognition procedure applied to image (a). The image is brighter in the middle because the background is not yet subtracted.

\section{Discussion}

The erythrocytes shape is strongly influenced by the sample preparation and experimental protocols. In this work, we made dry smears placing the cells on a flat 
glass surface. The cell concentration was low enough so that it was possible to isolate separately the single cells. However, as one can see from the Function 4 in Fig. 5 the obtained shape changes the visibility value critically. We have to follow such procedure of blood preparation in order to be closer to routine medical blood test in which a dry smear is analyzed using optical microscope. From this point of view, our calculations are far from ideal model but close to medical reality.

In principle, other protocols of blood smear preparation are available for the same purposes. For example, one can add glutaraldehyde into the blood probe and fix the erythrocyte shape, see Ref. [12] for more details. Being in normal conditions, the red cells take the shape of a biconcave disk, and glutaraldehyde will preserve this shape even when the erythrocytes dry up on the glass smear. For the cells prepared in such way, the method becomes much more stable as the main unknown parameter is fixed in advance. However, even in this case, the cells may have different shapes due to some blood diseases. Given such sample, our method should also show decreased value of the diffraction pattern visibility. Thus, the researchers should take into account that the visibility function reflects not only the dependence on RDW but also on the distribution of the cells in shape. To clarify this aspect by strict formulas, additional research is currently undertaken.

Note that placing the cells in a transparent liquid, generally leads to that the cells shape features make lower influence on the diffraction pattern. However, when working with liquid samples one has to take care about fluid evaporation in the sample and additional forces acting on blood cells. These forces may again change cells shapes, which should be taken into account in model calculations in laser diffractometry technique.

Most often in laser diffractometry, the small particles are placed into some liquid in a cuvette that leads to random orientations of the particles in space. In this case, results are still largely dependent on the cells shape as the shape is not uniform in all directions.

Whatever protocol of blood sample preparation one chooses, the basic requirement is that the results of laser diffractometry method may only be compared with the ones, achieved by using the same protocol. As described above, different procedures of blood sample preparation lead to unique typical distortions of the cells shapes and the visibility changes according to them.

\section{Conclusion}

Geometrical 3D models of human red blood cells in a dry smear were obtained experimentally using hyperspectral holography method. Corresponding diffraction patterns in the far field diffraction zone were calculated. Visibility values of the diffraction patterns were obtained in cases of low and high red cell size distribution width. The results revealed that the visibility is influenced by the cells shapes. Namely, the visibility is always lower when using the model of real cells shape to compare with the case of ideal symmetric shapes. However, the visibility of the diffraction pattern still can be used to assess the red cell distribution width in clinical practice.

Fig. 5 shows four calculated functions representing the main result. The visibility values are plotted along the vertical axis. The horizontal axis corresponds to the values of RDW denoted by $\delta_{R}$.

Function 1 shows the dependence of visibility on RDW in case when all the cells have an ideal circle base and ideal flat surface. We also assumed that there is no speckle structure in the diffraction pattern in case of Function 1. That is why the function is ideally monotonic. This function was first calculated in Ref. [5]. The Function 2 was calculated in the same conditions as Function 1 but the speckle structure in the diffraction pattern was taken into account. One can see that the Functions 1 and 2 are very close to each other within a small error space of about $5 \%$. This means that randomness of cells coordinates does not make too big impact into our model.

Function 3 represents the case when the shape of the cells was an ideal biconcave disk. Here, the visibility is not 1 even for very low values of RDW. Note that this figure was calculated when the red cells were in the air medium, thus their relative refractive index was 1.4 , that is a high value. If the cells were in the transparent liquid medium like water, then Function 3 would be very close to the Functions 1 and 2. This means that the model of the cells shape acts together with optical parameters of the medium in which the cells are located.

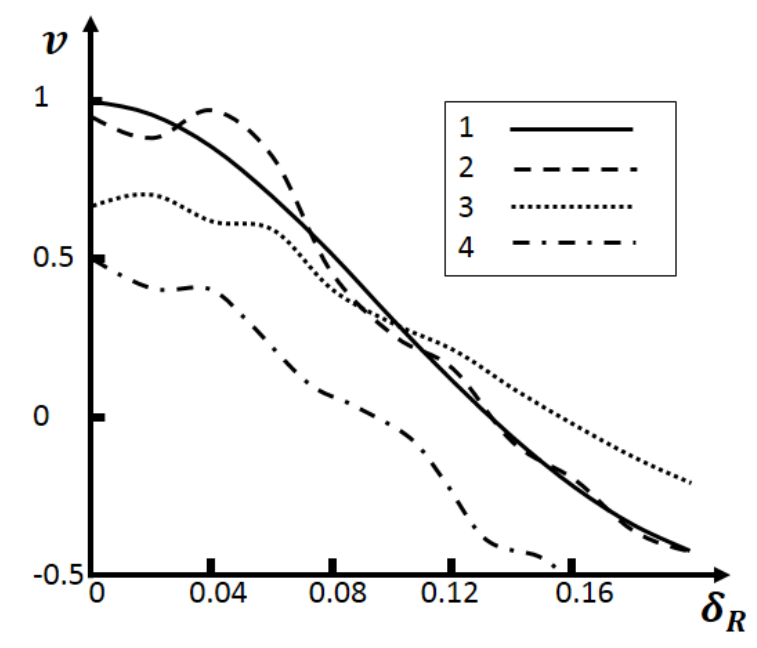

Fig. 5 Dependency of visibility $v$ on RDW $\delta_{R}$ in the cases of 4 different assumptions. 1 - ideal circular cylinders with no speckles, 2 - ideal circular cylinders with speckles, 3 - ideal biconcave disks with speckles, 4 - real 3D models of cells obtained experimentally with speckles.

To calculate the Function 4, we assumed that the cells are described by natural 3D geometrical models taken from the HH experiment as shown in Fig. 4. Cells of 3 typical images were taken as initial data. They were stretched, rotated and located randomly on the plane. One can see from Fig. 5 that taking into account the natural shape we significantly reduce the visibility. 
However, the calculated visibility still decreases with $\delta_{R}$ becoming larger. This further leads to a conclusion that the method of laser diffractometry is still applicable in case of natural dry erythrocytes shapes.

\section{Disclosures}

All authors declare that there is no conflict of interests in this paper.

\section{Acknowledgments}

This work was supported by RFBR grant № 17-2903507-ofi-m. Authors acknowledge the help of Dr. Alexei N. Semenov in preparing the blood smears and Prof. Sergey Yu. Nikitin for useful discussions.

\section{References}

1. K. V. Patel, L. Ferrucci, W. B. Ershler, D. L. Longo, and J. M. Guralnik, "Red blood cell distribution width and the risk of death in middle-aged and older adults," Archives of Internal Medicine 169(5), 515-523 (2009).

2. G. I. Kozinets, V.A. Makarova, The study of the blood system in clinical practice, Triada-X, Moscow (1997) [in Russian].

3. D. L. Black, M. Q. McQuay, and M. P. Bonin, "Laser-based techniques for particle-size measurement: a review of sizing methods and their industrial applications," Progress in energy and combustion science 22(3), 267-306 (1996).

4. W. M. Farmer, "Measurement of particle size, number density, and velocity using a laser interferometer," Applied Optics 11(11), 2603-2612 (1972).

5. S. Yu. Nikitin, A. E. Lugovtsov, A. V. Priezzhev, and V. D. Ustinov, "Relation between the diffraction pattern visibility and dispersion of particle sizes in an ektacytometer," Quantum Electronics 41(9), 843-846 (2011).

6. Yu. S. Yurchuk, V. D. Ustinov, S. Yu. Nikitin, and A. V. Priezzhev, "Scattering of a laser beam on a wet blood smear and measurement of red cell size distribution," Quantum Electronics 46(6), 515-520 (2016).

7. G. S. Kalenkov, S. G. Kalenkov, and A. E. Shtan'ko, "Hyperspectral holographic Fourier-microscopy," Quantum Electronics 45(4), 333-338 (2015).

8. S. G. Kalenkov, G. S. Kalenkov, and A. E. Shtanko, "Hyperspectral holography: an alternative application of the Fourier transform spectrometer," Journal of the Optical Society of America B 34(5), B49-B55 (2017).

9. A. I. Yusipovich, E. Yu. Parshina, N. Yu. Brysgalova, A. R. Brazhe, N. A. Brazhe, A. G. Lomakin, G. G. Levin, and G. V. Maksimov, "Laser interference microscopy in erythrocyte study," Journal of Applied Physics 105(10), 102037 (2009).

10. G. G. Levin, G. N. Vishnyakov, and V. L. Minaev, “An automated interference microscope for measuring dynamic objects," Instruments and Experimental Techniques 56(6), 686-690 (2013).

11. S. Yu. Nikitin, A. V. Priezzhev, and A. E. Lugovtsov, "Laser diffraction by the erythrocytes and deformability measurements," Chapter 6 in Advanced optical flow cytometry: methods and disease diagnoses, V. Tuchin (Ed.), Wiley-VCH, Weinheim, Germany, 133-154 (2011).

12. I. Dulińska, M. Targosz, W. Strojny, M. Lekka, P. Czuba, W. Balwierz, and M. Szymoński, "Stiffness of normal and pathological erythrocytes studied by means of atomic force microscopy," Journal of Biochemical and Biophysical Methods 66(1-3), 1-11 (2006). 